\title{
Method of Successive Approximations for a Fluid Structure Interaction Problem
}

\author{
Aliou Gueye Sow, Ibrahima Mbaye \\ Department of Mathematics, University of Thies, Thies, Senegal \\ Email: aliounesow84@yahoo.fr
}

Received 17 May 2014; revised 25 June 2014; accepted 9 July 2014

Copyright (C) 2014 by authors and Scientific Research Publishing Inc.

This work is licensed under the Creative Commons Attribution International License (CC BY). http://creativecommons.org/licenses/by/4.0/

(c) (i) Open Access

\section{Abstract}

In this paper, we present a method for solving coupled problem. This method is mainly based on the successive approximations method. The external force acting on the structure is replaced by $\lambda=p\left(x_{1}, H+u\left(x_{1}, \lambda\right)\right)$. Then we have a nonlinear equation of unknown $\lambda$ to solve by successive approximations method. By this method, we obtain easily the analytic expression of the displacement. In addition, good results are obtained with only a few iterations.

\section{Keywords}

\section{Beam Equation, Stokes Equation, Finite Elements Method, Successive Approximations Method}

\section{Introduction}

Problem involved in fluid structure interactions occurs in a wide variety of engineering problem and therefore has attracted the interest of many investigations from different engineering disciplines. As a result, much effort has gone into the development of general computational method for fluid structure system by Osses, Fernandez, quarteroni, Blouza, Mbaye [1]-[7].

In this paper, successive approximations method is applied to solve a fluid-structure interaction problem. We replace the external force acting on the interface between fluid and structure by $\lambda=p\left(x_{1}, H+u\left(x_{1}, \lambda\right)\right)$.

Then we introduce a nonlinear equation to solve by successive approximations such that the coupled problem is achievable.

By this method, we obtain good approximate solutions. In addition, the analytic solution of beam equation can be computed easily.

The fluid is modeled by two dimensional Stokes equations for steady flow and the structure is represented by 
the one dimensional beam equation.

\section{Presentation of the Problem}

We denote by $\Omega_{F}$ the two dimensional domain occupied by the fluid, $\Gamma_{u}$ the elastic interface between fluid and structure and $\Gamma=\sum_{1} \cup \Sigma_{2} \cup \Sigma_{3}$ be the remaining external boundaries of the fluid as depicted in Figure 1.

$\Omega_{0}=[0, L] \times[0, H]$ defines the reference domain.

\section{Governing Equations}

\subsection{Structure Equation}

We start from the simple equation that governs the structure. The simplified beam equation is:

$$
\begin{gathered}
D u^{(4)}\left(x_{1}\right)=f^{S}\left(x_{1}\right) \text { for } 0<x_{1}<L, \\
u(0)=u(L)=u^{(1)}(0)=u^{(1)}(L)=0 .
\end{gathered}
$$

where $u$ is the displacement of the structure, $f^{s}$ is the external force of the structure, $D=\frac{E h^{3}}{12}, E$ is the young modulus, $h$ is the thickness of the structure This equation is good representation of the structure for small deformation.

\subsection{Fluid Equation}

We suppose that the fluid is governing by the Stokes equations for steady flow in $\Omega_{F}$ :

$$
\begin{gathered}
-\mu \Delta v+\nabla p=f^{F} \text { in } \Omega_{\mathrm{F}}, \\
\nabla \cdot v=0 \text { in } \Omega_{\mathrm{F}}, \\
v=g \text { on } \Sigma_{1}, \\
v \cdot n=0 \text { on } \Sigma_{2}, \\
\frac{\partial v_{1}}{\partial y}=0 \quad \text { on } \Sigma_{2}, \\
-p I n+\frac{\partial v}{\partial n}=0 \quad \text { on } \Sigma_{3}, \\
v=0 \quad \text { on } \Gamma_{u},
\end{gathered}
$$

where $v$ denotes the fluid velocity, $p$ denotes the pressure, $f^{F}$ denotes the volume force of the fluid, $\mu$ the viscosity of the fluid and $g$ denotes the inflow velocity profile of the fluid, $n$ is the unit outward normal vector, $I$ is the identity matrix, on the symmetric axis $\Sigma_{2}$ we have the non-penetration condition $v \cdot n=v_{2}=0$.

\section{Formulation of Coupled Problem}

The problem is to find $u, v$ and $p$ such that:

$$
\begin{gathered}
D u^{(4)}\left(x_{1}\right)=p\left(x_{1}, H+u\left(x_{1}\right)\right) \text { for } 0<x_{1}<L \\
u(0)=u(L)=u^{(1)}(0)=u^{(1)}(L)=0, \\
-\mu \Delta v+\nabla p=f^{F} \quad \text { in } \quad \Omega_{\mathrm{F}}, \\
\nabla \cdot v=0 \quad \text { in } \quad \Omega_{\mathrm{F}}, \\
v=g \quad \text { on } \quad \Sigma_{1},
\end{gathered}
$$




$$
\begin{gathered}
v \cdot n=0 \quad \text { on } \quad \Sigma_{2} \\
\frac{\partial v_{1}}{\partial y}=0 \quad \text { on } \quad \Sigma_{2}, \\
-p I n+\frac{\partial v}{\partial n}=0 \quad \text { on } \quad \Sigma_{3}, \\
v=0 \quad \text { on } \Gamma_{u},
\end{gathered}
$$

We have a fluid structure interaction problem. The domain of the fluid depends on the displacement and the displacement depends on the velocity and the pressure of the fluid.

\section{Successive Approximations Method}

We assume that $\lambda=p\left(x_{1}, H+u\left(x_{1}, \lambda\right)\right)$. Corresponding to each $\lambda$, we consider the coupled problem:

$$
\begin{gathered}
D u^{(4)}\left(x_{1}\right)=\lambda \text { for } \quad 0<x_{1}<L \\
u(0)=u(L)=u^{(1)}(0)=u^{(1)}(L)=0, \\
-\mu \Delta v+\nabla p=f^{F} \quad \text { in } \quad \Omega_{\mathrm{F}}, \\
\nabla \cdot v=0 \quad \text { in } \quad \Omega_{\mathrm{F}}, \\
v=g \quad \text { on } \quad \Sigma_{1}, \\
v \cdot n=0 \quad \text { on } \quad \Sigma_{2}, \\
\frac{\partial v_{1}}{\partial y}=0 \quad \text { on } \quad \Sigma_{2}, \\
-p I n+\frac{\partial v}{\partial n}=0 \quad \text { on } \quad \Sigma_{3}, \\
v=0 \quad \text { on } \quad \Gamma_{u},
\end{gathered}
$$

To solve this coupled problem, we need to solve a nonlinear equation of unknown $\lambda$ define as: $\lambda=p\left(x_{1}, H+u\left(x_{1}, \lambda\right)\right)$ by the successive approximations method. Then we will find $u, v$ and $p$.

\section{Description of the Method}

The weak formulation of the fluid and the structure is given by Grandmont, Murea [8] [9].

We summarize step by step our computational method to find $\left(\lambda^{n}\right)$ such that:

$$
\begin{aligned}
& \lambda^{0}, \text { the initial value is done } \\
& \lambda^{n+1}=p\left(x_{1}, H+u\left(x_{1}, \lambda^{n}\right)\right),
\end{aligned}
$$

- Step 1: We give $\lambda^{0}$, the initial displacement and the fluid domain are compute.

- Step 2: We solve the Stokes equation by finite elements methods in the reference domain. We find $(v, p):=\left(v_{0}, p_{0}\right)$,

- Step 3: we fine $\lambda^{1}=p\left(x_{1}, H+u\left(x_{1}, \lambda^{0}\right)\right)$,

- Step 4: Do:

- $\lambda^{0}=\lambda^{1}$,

- compute $u\left(x_{1}, \lambda^{1}\right)$ and $\Omega_{F}\left(\lambda^{1}\right)$,

- solve the stokes equation in $\Omega_{F}\left(\lambda^{1}\right)$, we find

- $(v, p):=\left(v_{1}, p_{1}\right)$, 
- we compute $\lambda^{1}=p\left(x_{1}, H+u\left(x_{1}, \lambda^{0}\right)\right)$,

- While $\left(\left|\lambda^{1}-\lambda^{0}\right| \geq\right.$ tol $)$,

- Step 5: Give $\lambda, u,(v, p)$.

\section{Numerical Results}

For each $\lambda$, the analytic solution of the beam equations is

$$
u=\frac{\lambda}{D}\left(\frac{x^{4}}{24}-\frac{L x^{3}}{12}+\frac{L^{2} x^{2}}{24}\right) .
$$

We assume that the velocity on $\Sigma_{1}$ is $v=g=\left(v_{1}, v_{2}\right)$ such that $v_{1}\left(x_{1}, x_{2}\right)=30\left(1-x_{2}^{2} / H^{2}\right)$ and $v_{2}\left(x_{1}, x_{2}\right)=0$ for all $\left(x_{1}, x_{2}\right)$ in $\Sigma_{1}$ Murea [10].

The parameter values of the fluid and the structure are:

Parameter related to fluid: The fluid velocity is $\mu=0.035 \mathrm{~g} / \mathrm{cm} \cdot \mathrm{s}$, the fluid density is $\rho^{F}=1 \mathrm{~g} / \mathrm{cm}^{3}$, the channel length is $L=3 \mathrm{~cm}$, the channel width $H=0.5 \mathrm{~cm} \quad f^{F}=(0,0)$.

Parameter related to structure: The structure thickness $h=0.1 \mathrm{~cm}$, Young's modulus is $E=0.75 \times 10^{6} \mathrm{~g} / \mathrm{cm} \cdot \mathrm{s}^{2}, \quad$ tol $=10^{-4}$.

We use the $P 1 b$ Lagrange finite element to approach the velocities and $P 1$ Lagrange finite element is used to approach the pressure. FreeFem++ Hecht [11] is using for the numerical tests.

\section{Conclusion}

In this work, we applied successive approximations method to solve fluid structure interaction problem. This method gives good results when the displacement is small. After 11 iterations, we found a good approximate solution of the nonlinear equation and also we obtained the solution of coupled problem.

Table 1 and Figure 2 show that Error $=\left|\lambda^{1}-\lambda^{0}\right|$ decreases to zero when iterations increase. Figure 3 and Figure 4 display the behavior of the fluid flow and the pressure wave respectively after 11 .

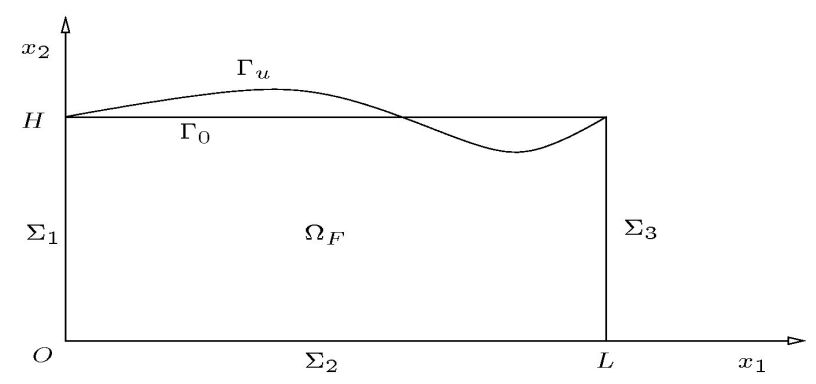

Figure 1. Sets appearing to the fluid structure problem.

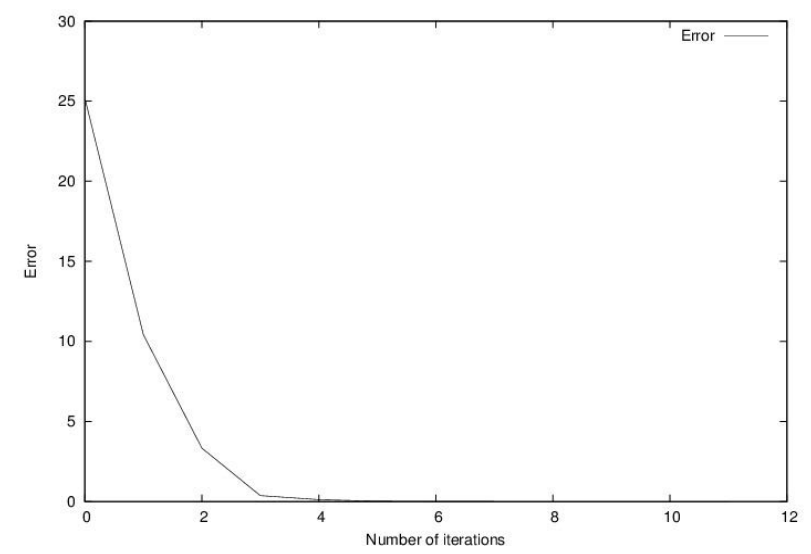

Figure 2. Error with respect to number iterations. 


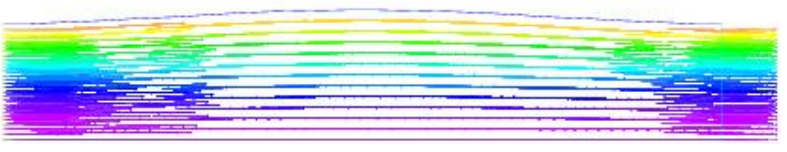

Figure 3. Fluid velocity and structure displacement.

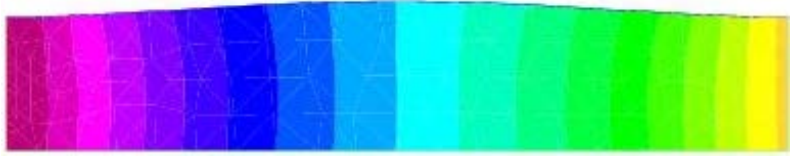

Figure 4. Fluid pressure.

Table 1. Different values of $\lambda$ after 11 iterations.

\begin{tabular}{cccc}
\hline$\lambda^{1}$ & $\lambda^{0}$ & Error $=\left|\lambda^{1}-\lambda^{0}\right|$ & Iterations \\
\hline 25.1986 & 0 & 25.1986 & 0 \\
14.776 & 25.1986 & 10.4227 & 1 \\
18.1165 & 14.776 & 3.34049 & 2 \\
17.7406 & 18.1165 & 0.375886 & 3 \\
17.8601 & 17.7406 & 0.119541 & 5 \\
17.8368 & 17.8601 & 0.0233231 & 6 \\
17.8456 & 17.8368 & 0.0087835 & 7 \\
17.8423 & 17.8456 & 0.00330984 & 8 \\
17.8435 & 17.8423 & 0.00124704 & 9 \\
17.843 & 17.8435 & 0.000468658 & 10 \\
17.8432 & 17.843 & 0.000173383 & 11 \\
17.8432 & 17.8432 & $6.21913 \mathrm{e}-005$ & \\
\hline
\end{tabular}

In a forthcoming work, we will be showed the theoretical convergence of $\lambda^{n+1}=p\left(x_{1}, H+u\left(x_{1}, \lambda^{n}\right)\right)$ and also successive approximations method will be used to solve an unsteady fluid structure interaction problem.

\section{References}

[1] Osses, A. and Puel, J.P. (2000) Approximate Controllability for a Linear Model of Fluid Structure Interactiont. ESAIM Control, Optimization and Calculus of Variation, 4, Article ID: 4976513.

[2] Fernandez, M.A. and Moubachir, M. (2003) And Exact Block Newton Algorithm for Solving Fluid Structure Interaction Problem. Compte rendu de l'académie des sciences, paris, Serie I, 336, 681-686.

[3] Fernandez, M.A. and Moubachir, M. (2005) A Newton Method Using Exact Jacobians for Solving Fluid-Structure Coupling. Computers and Structures, 83, 127-142.

[4] Quateroni, A., Tuveri, M. and Veneziani, A. (2000) Computational Vascular Fluid Dynamics, Problem Models and Methods. Computing and Visualization in Science, 2, 163-197. http://dx.doi.org/10.1007/s007910050039

[5] Blouza, A., Dumas, L. and Mbaye, I. (2008) Multiobjective Optimization of a Stent in a Fluid-Structure Context. Proceedings of Genetic and Evolutionary Computation Conference (GECCO), 2056-2060. http://dx.doi.org/10.1145/1388969.1389021

[6] Mbaye, I. (2012) Controllability Approach for a Fluid-Structure Interaction Problem. Applied Mathematics, 3, $213-216$. http://dx.doi.org/10.4236/am.2012.33034

[7] Mbaye, I. and Murea, C. (2008) Numerical Procedure with Analytic Derivative for Unsteady Fluid-Structure Interaction. Communications in Numerical Methods in Engineering, 24, 1257-1275.

[8] Grandmont, C. (1998) Existence et Unicité de Solution d'un Problème de Couplage Fluide-Structure Bidimensionnel 
stationnaire. Compte rendu de l'académie des sciences, Paris.

[9] Murea, C. and Maday, C. (1996) Existence of an Optimal Control for a Nonlinear Fluid Cable Interaction Problem. Rapport de Recherche CEMRACS, C.I.R.M., Luminy.

[10] Murea, C. (2005) The BFGS Algorithm for Nonlinear Least Squares Problem Arising from Blood Flow in Arteries. Computers Mathematics with Applications, 49, 171-186. http://dx.doi.org/10.1016/j.camwa.2004.11.002

[11] Hecht, F. and Pironneau, O. (2003) A Finite Element Software for PDE FreeFem++. www.freefem.org/ff++/ 
Scientific Research Publishing (SCIRP) is one of the largest Open Access journal publishers. It is currently publishing more than 200 open access, online, peer-reviewed journals covering a wide range of academic disciplines. SCIRP serves the worldwide academic communities and contributes to the progress and application of science with its publication.

Other selected journals from SCIRP are listed as below. Submit your manuscript to us via either submit@scirp.org or Online Submission Portal.
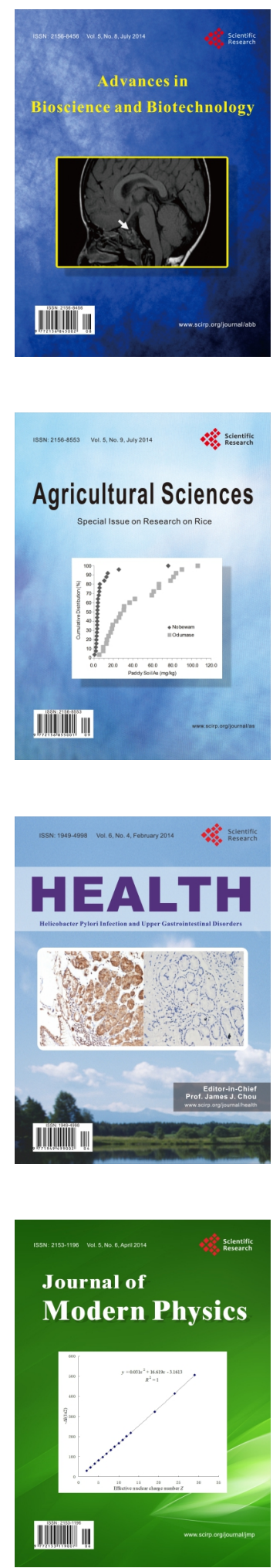
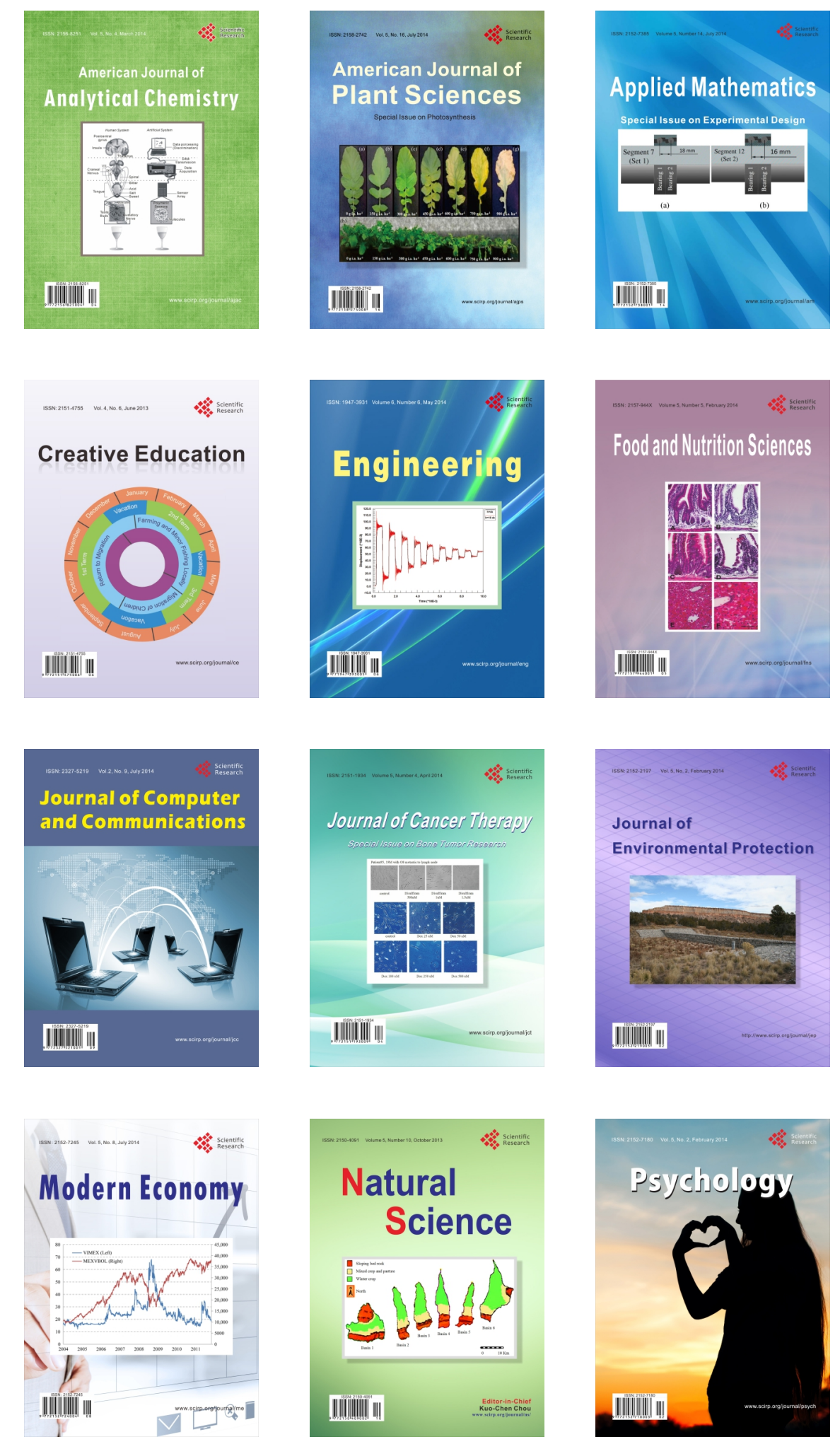